\title{
A New Production Platform for Authoring Object-based Multiscreen TV Viewing Experiences
}

\author{
Jie Li \\ CWI \\ Amsterdam, Netherlands \\ jie.li@cwi.nl \\ Jack Jansen \\ CWI \\ Amsterdam, Netherlands \\ jack.jansen@cwi.nl
}

\author{
Thomas Röggla \\ CWI \\ Amsterdam, Netherlands \\ t.roggla@cwi.nl
}

\author{
Maxine Glancy \\ BBC R\&D \\ Manchester, United Kingdom \\ maxine.glancy@bbc.co.uk
}

\author{
Pablo Cesar \\ CWI \& TU Delft \\ Amsterdam, Netherlands \\ p.s.cesar@cwi.nl
}

\begin{abstract}
Multiscreen TV viewing refers to a spectrum of media productions that can be watched on TV screens and companion screens (e.g., smartphones and tablets). TV production companies are now promoting an interactive and engaging way of viewing $\mathrm{TV}$ by offering tailored applications for TV programs. However, viewers are demotivated to install dozens of applications and switch between them. This is one of the obstacles that hinder companion screen applications from reaching mass audiences. To solve this, TV production companies need a standard process for producing multiscreen content, allowing viewers to follow all kinds of programs in one single application. This paper proposes a new object-based production platform for authoring programs for multiscreen. The platform consists of two parts: the preproduction tool and the live editing tool. To evaluate whether the proposed workflow is appropriate, validation interviews were conducted with professionals in the TV broadcasting industry. The professionals were positive about the proposed new workflow, indicating that the platform allows for preparations at the preproduction stage and reduces the workload during the live broadcasting. They see as well its potential to adapt to the current production workflow.
\end{abstract}

\section{Author Keywords}

Multiscreen TV viewing; Object-based broadcasting; Production tools; Graphical interface design.

Permission to make digital or hard copies of all or part of this work for personal or classroom use is granted without fee provided that copies are not made or distributed for profit or commercial advantage and that copies bear this notice and the full citation on the first page. Copyrights for components of this work owned by others than ACM must be honored. Abstracting with credit is permitted. To copy otherwise, or republish, to post on servers or to redistribute to lists, requires prior specific permission and/or a fee. Request permissions from Permissions@acm.org.

TVX '18, June 26-28, 2018, SEOUL, Republic of Korea

(C) 2018 Association for Computing Machinery.

ACM ISBN 978-1-4503-5115-7/18/06 ..\$15.00

https://doi.org/10.1145/3210825.3210834

\section{ACM Classification Keywords}

- Human-centered computing User studies • Humancentered computing Field studies

\section{INTRODUCTION}

Traditional studies on TV viewing considered viewers' relaxation and passivity as important factors that contribute to their enjoyment [7]. Lately, with the prevalence of smart companion devices (i.e., smartphones and tablets), it is common to see that people's attention is easily distracted from the TV screen to companion screens [19, 21]. TV viewing is no longer a lean-back experience. The media industry foresees the potential of multiscreen. They are promoting an interactive and engaging way of viewing TV by offering auxiliary content and interactive functions on companion screens $[1,4,8,18,20,25]$. These applications were all tailored for specific TV programs, becoming impractical for viewers to install and switch between dozens of inconsistent ones. Much efforts have been invested in companion applications, but hardly any of them reached mass audiences [5].

Conventional ways of TV production are well established. The processes for preproduction, live and recorded broadcasting to TV screens is standardized [23]. However, there is no standard process for producing companion screen content. Instead of developing applications in an ad-hoc manner, Geerts et al. [10] suggested providing a single application with which viewers could follow all the companion screen shows of a broadcaster. In a previous study [16], a preproduction tool was designed for authoring TV programs for multiscreen. The tool was evaluated by professionals in the TV broadcasting industry. They indicated that preproduction for live broadcasting is radically different from that for recorded broadcasting. Live broadcasting is time critical and always has a certain unpredictability. Even for broadcasting to TV screens only, it is a challenge for the director and the production team to react to live events or search databases for new materials [12, 15]. The professionals suggested that only a preproduction tool is not sufficient to guarantee successful broadcasting of 
multiscreen content. An integrated platform that includes both preproduction and live broadcasting was proposed [16].

The focus of this paper is on an object-based production approach [2] for authoring multiscreen content. The goal is to design a live editing tool, together with a preproduction tool, to form an integrated platform that supports the authoring and broadcasting of multiscreen content. To do so, the first step is to understand the current workflows of live broadcasting. A field study was conducted at a live OB (Outside Broadcasting) truck covering the MotoGP 2017 race at Silverstone. Due to the rapid pace of MotoGP races and various types of live events (e.g., crashes, overtakes), this field study offered us a good opportunity to observe the intense workflow and rapid decisions needed for live broadcasting. Afterwards, a live triggering tool and a proposed workflow for authoring and broadcasting multiscreen content were designed. Finally, validation interviews with professionals in the broadcasting industry were conducted with three research questions:

1) Is the proposed workflow for multiscreen production appropriate? Does it fit into the current production workflow?

2) Are the tools (preproduction \& live editing) adequately designed? What are the suggestions from the professionals for improvement?

3) Who is the right person in the production team to use such new tools?

The paper is organized as follows. First, related work introduces several object-based production concepts and multiscreen viewing experiences. It also summarizes a typical production workflow, highlighting the differences and challenges of live and recorded broadcasting. Next, the background section recalls the results of one previous study and the design of a preproduction tool. Then, we describe the field study at MotoGP 2017 (Silverstone) and visualize the current workflow of live broadcasting for a MotoGP racing event. Based on the insights from the field study, a live editing tool is designed. In the end, the validation interviews of the production platform and the results are presented. We conclude that the workflow proposed by the platform was appreciated by the professionals. They indicated that the user interfaces of the platform are straightforwardly designed, so they can be easily mastered by the production team. The platform allows preparations at the preproduction stage and reduces the workload during live broadcasting. They foresaw the potential of the platform in producing multiscreen contents and provided feedback to improve the interface design. They suggested that the current production team needs to hire additional staff to operate the platform. The platform can run in parallel with the existing production workflows. However, it might require time and effort to get the production team to accept and trust a new system.

\section{RELATED WORK}

This section describes some recent research about objectbased broadcasting and production workflows.

\section{Object-based Broadcasting}

The concept of object-based media is not new. The field of computer games is advanced in applying this approach, allowing game players to follow customized playing modes and to fully interact with the game world. What is an objectbased production process? Here, "object" refers to different media units that are used to make a TV program. The objectbased approach involves breaking down a program into separate content objects, typically including graphics, audio, video, background music, dialogues, subtitles, sound/visual effects, etc., and describing how they can be rearranged. With this approach, a program can be adapted to fulfill the needs of different individual viewers $[15,26]$.

Some recent studies have explored object-based production in different use cases. Cox et al. [6] developed a personalized interactive cooking application called CAKE. CAKE supports an interactive dialogue between a viewer and a cooking show. CAKE can integrate multiple recipes selected by the viewer and automatically generate a step-by-step cooking plan. In this way, the show is adjustable according to the viewer's pace. Squeezebox developed by BBC Research \& Development [3] is an object-based tool that enables rapid re-editing the duration of the content. Squeezebox can automatically analyze and segment the footage into individual shots. The production team can mark up the priority of each shot, determining whether the footage will be cut or preserved as the duration is reduced. Most recently, Puentes et al. [22] developed a flexible visual authoring tool. By dragging and dropping static or dynamic "components" (e.g., texts, images, videos, audio, fonts, etc.) into "containers" (e.g., defined regions on the screens). Developers and designers become time-and-cost-efficient in creating interactive TV applications.

As a summary, Figure 1 compares the traditional linear TV program broadcasting and the object-based broadcasting. The two broadcasting approaches both start with preparing all content objects. The linear broadcasting transmits the same program to every viewer, resulting in a passive and lean-back viewing experience. It remains difficult for content providers to make different versions of the program to fit different screen formats, audiences and environments. In contrast, object-based broadcasting transmits content objects independently to viewers along with the metadata describing how they should be assembled. The devices controlled by the viewers optimally assemble the content objects $[2,17,26]$.

The object-based production platform proposed in this paper consists of a collection of interactive media units configured to work together to deliver the look and feel of a single tool. This collection is called Distributed Media Application (DMApp) [15]. Reusable media units (DMApp Components) are assembled during the preproduction to create coherent multiscreen experiences and be aired during the live broadcasting. The set of DMApp components are actually a set of content objects as shown in Figure 1, which can also be configured to have some interactive and novel 
functions, such as "like" widget (for expressing "like" preference), real-time video chat or text chat, which can stimulate viewers' engagement. The DMApp components are one of the essential elements of the production tool design.

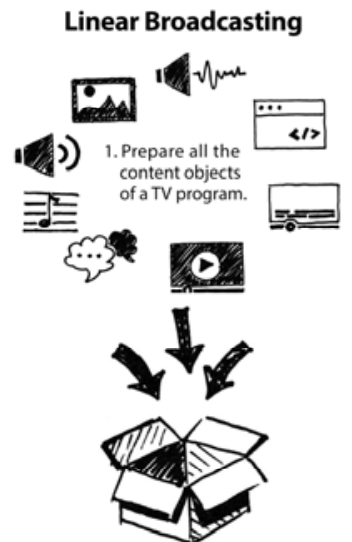

2. All the content objects are composited and turned into a linear program during the transmitted to every viewer.

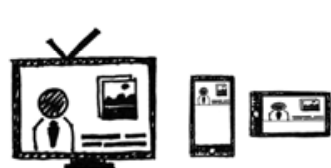

3. The same program is displayed on all kinds of devices, which can neither be tailored to the viewers' contexts, nor allow viewers to interact meaningfully.

Figure 1. Compare the traditional linear broadcasting with the object-based broadcasting.

\section{Typical Television Production Workflow}

A production workflow typically consists of three phases, namely preproduction, production and postproduction [23]. Pre-production contains all kinds of preparations for the production. The main activity at this phase is to break down the script into individual scenes. All the media content (audio, video, graphics, visual and sound effects, etc.) are prepared accordingly. The primary task of the production phase is to film live shows or raw footages for postproduction. The goal of postproduction is to make precise edits and to select the best shots for broadcasting. However, for live broadcasting, the time for postproduction is limited. Instead of extensive postproduction, what the director does is called "live editing", which involves quickly mixing live camera feeds, selecting and editing some shots to prevent undesirable materials being aired. For recorded broadcasting, there can be iterations between postproduction and filming. The director may request to shoot extra video footages or reshoot some unsatisfying scenes. Due to the time limit and unpredictability of the live broadcasting, live editing is the most overwhelming task for a TV production team, even for broadcasting only to TV screens. Figure 2 shows a summary of the main tasks of the three phases. We believe that current working practices will need to evolve to accommodate the requirements of multiscreen production.

\section{BACKGROUND}

In a previous study, semi-structured interviews with professionals in the TV production industry were conducted to collect requirements concerning the tool design for multiscreen TV production [17]. According to the requirements, four wireframe concepts of a preproduction tool were designed. Professionals from the broadcasting industry were invited to evaluate these concepts. One of the concepts was selected and redesigned based on the consensus of the professionals [16].

The professionals specifically pointed out that the preproduction tool should differentiate the authoring for live and recorded broadcasting, due to the time limitation and unpredictability of live broadcasting. During live broadcasting, the whole team works like a symphony orchestra. Mistakes do happen, but every effort is made to minimize their occurrence. One of the professionals recommended a simplified live triggering tool to reduce the burden. The professionals also advised that the production team should be able to quickly build up events during live broadcasting, using data packages such as the drivers' profiles, live statistics, and camera feeds. Once live events happen (e.g., riders crash and overtake in a motorcycle race), the live broadcasting team needs to quickly trigger replays of the events. Customized templates should be adopted in this situation rather than to create things from scratch.

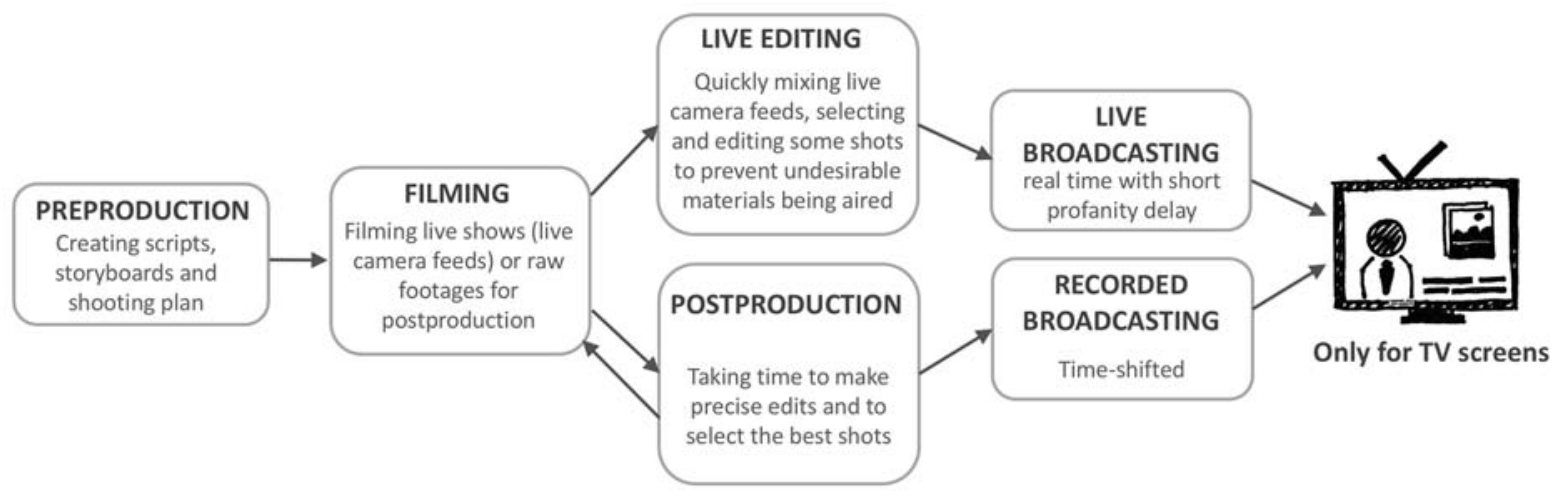

Figure 2. A typical television production workflow. Main tasks of each phase are described. 

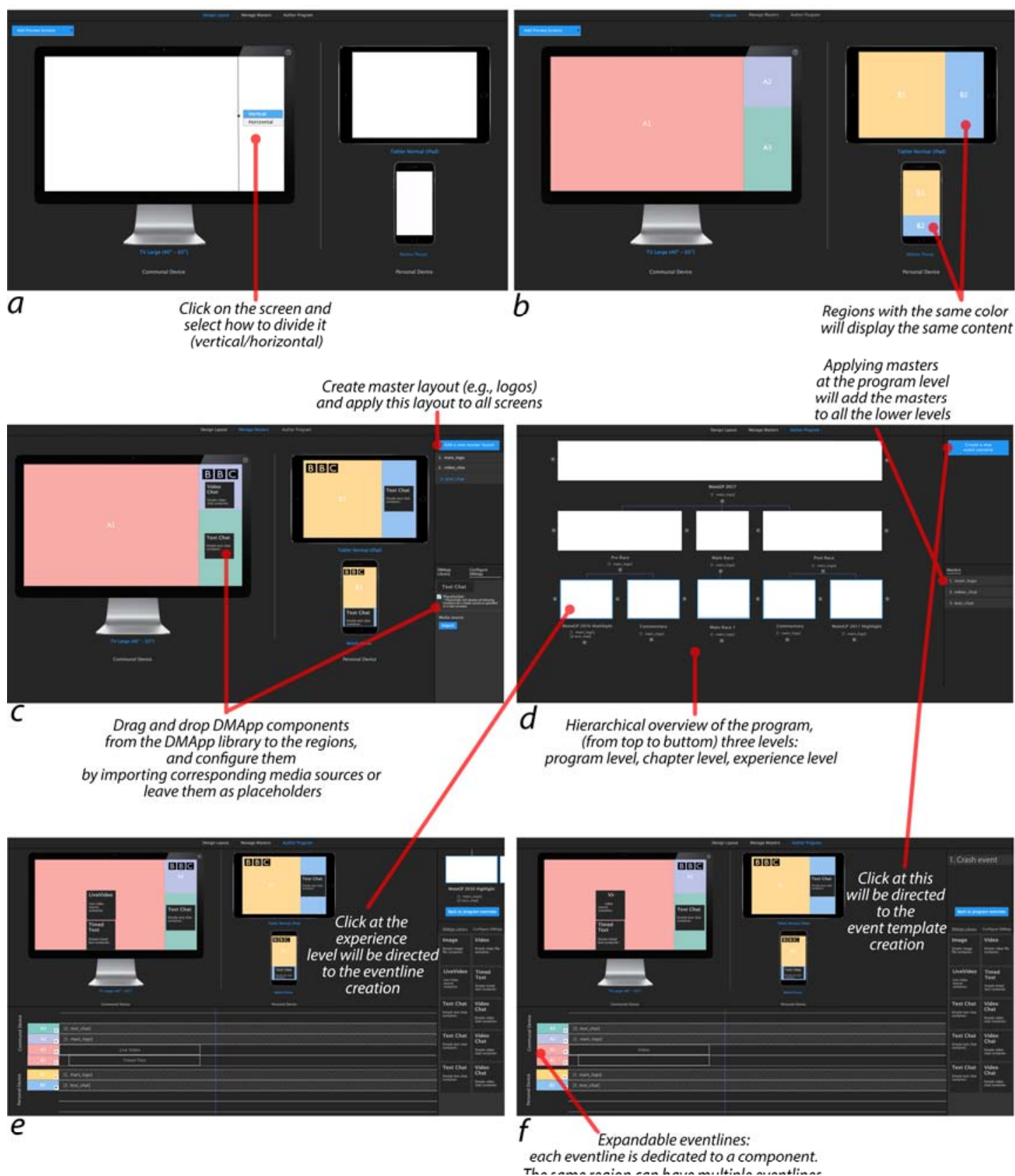

Figure 3. The advanced mode of layout design: (a) Click on the screen to divide it vertically or horizontally; (b) Label the regions with names and colors. Regions with the same color will display the same content. The eventline and event templates creation: (c) Create master layout, (d) Hierarchical overview of the program, (e) Create and edit content at the experience level, and (f) Create and edit content for live events.

Figure 3 shows featured screenshots of the re-designed preproduction tool, based on the feedback from the professionals [16]. Creating templates for live events is also included in the preproduction tool. Figure $3 \mathrm{a}$ and $3 \mathrm{~b}$ illustrate the layout design mechanism in the advanced mode. The easy mode of layout design is to incorporate existing brand templates. The advanced mode allows program authors to flexibly divide regions on screens and label the regions with different colors. Regions with the same color will display the same content. For instance, the yellow and blue regions on the screens of the tablet and the phone. Figure $3 \mathrm{c}-3 \mathrm{f}$ depicts the eventline and event templates creation. Figure $3 \mathrm{c}$ shows the way to create a master layout, for example, a master layout of the "main logo". Figure 3d exhibits a three-level hierarchical overview of the program. From top to bottom, these levels are the program level, the chapter level and the experience level. Once created, the master layout can be applied at the program level. Then, the same master will be automatically added at the chapter level and the experience level. The master layout reduces repetitive work. Figure 3e 
and $3 \mathrm{f}$ are very similar, both including previews on multiple screens, eventlines and a library of DMApp components. Clicking on one of the documents at the experience level will direct to the "interface" shown in Figure 3e, where experience can be created, previewed and edited. The interface shown in Figure $3 f$ has the same function, but for creating, previewing and editing live events, such as a crash at a motorcycle race or a goal in a football match.

As suggested by professionals, apart from the preproduction tool, a live editing tool should be designed to reduce the workload of live broadcasting, especially when broadcasting for multiscreen. All kinds of anticipated events and DMApp components can be prepared in the preproduction tool. With the live editing tool, the director can trigger the prepared events and components to multiscreen at adequate moments and remove them when the program requires. To design such a live tool, a field study was conducted at MotoGP 2017, to observe the live broadcasting workflow at an outside broadcasting $(\mathrm{OB})$ truck. The next section will present the methods and results of the MotoGP study.

\section{A FIELD STUDY AT MOTOGP}

The goal of the study is to understand the workflow and challenges of live broadcasting. We chose to conduct the study at a representative event: the MotoGP 2017 racing event. MotoGP is the premier class of motorcycle racing events and is overwhelming for broadcasters due to the rapid pace of the races and unpredictable live events (overtake, crash, etc.). Due to the complexity, the MotoGP live broadcasting workflow adequately represents live sports broadcasting.

The commercial and television rights of the MotoGP have been exclusively held by Dorna, an international sports management and media company. Dorna provides the "World Feed" (i.e., IPF=International Program Feed) of the MotoGP racing to all other broadcasters worldwide. North One Television is the production company responsible for producing the presentation of Dorna's IPF for all the BT Sport's MotoGP broadcasts including the British MotoGP at Silverstone. North One Television hosted the field study, which was conducted in their OB truck gallery, their OB truck has standard setups for on-site broadcasting.

\section{Methods}

Four researchers participated in the three-day field study, from August 25 to 27, 2017, following a field data gathering technique called contextual inquiry [14]. When everyday work becomes habitual and unconscious, people are usually unable to articulate it. Contextual inquiry reveals these unconscious and tacit aspects of work. It guides researchers and designers to stay with people at their working place and talking with them about their work while observing them [14]. Due to the intense live OB broadcasting, the researchers were not allowed to talk to the staff during their work.

The first two days were the MotoGP warm-ups, and the third day was the official race. The four researchers were divided into two groups, which took turns to do the observations, swapping every two hours. A production manager who was not working in the OB truck was sitting together with the researchers during the first 30 minutes of the observation, assisting the researchers in understanding the set-ups, roles and workflows in the OB truck. Researchers were allowed to ask the production manager questions in a low voice. The OB live broadcasting of the warm-up races and the official race were observed, and the conversations between the staff were noted. The official race was video recorded.

\section{Data analysis}

The notes of the researchers and the video recordings were analyzed right after the field study. The notes of the four researchers were combined, with many overlapped content such as the OB truck set-ups, the transcripts of the conversations between the staff, and the workflow descriptions. The researchers discussed and rationalized the overlapped content, reaching a shared understanding of challenges in live broadcasting. The video recordings and conversations between staff were further analyzed to reveal the workflow.

\section{Results}

The results first illustrate the set-ups and roles of people who participated in the live broadcasting. Then, it visualizes the workflow and explains what is crucial for quickly reacting to live events.

\section{The $O B$ truck set-ups and roles of staff}

In the OB truck, staff were arranged into two rows as shown in Figure 4. Apart from the OB truck, three InVision teams were working near the racing track, which consist of a presenter, a producer, and a cameraman. The director or the producer at the OB truck gallery can communicate with the InVision teams and send them instructions about what to do (e.g., instruct the presenter to talk about a particular topic and the cameraman to shoot certain scenes).

The role and tasks of the $\mathrm{OB}$ truck staff are described as follows:

1) Assistant producer. Keep up with the schedule, counting down with a stopwatch.

2) Producer. Decide together with the director, and inform the whole team about, a sequence of content that will be presented next, typically during a break. Work together with the production coordinator about what replay clips should be made.

3) Director. Decide the look and feel of the program. Instruct the whole team. Communicate with the presenters and the cameramen.

4) Graphic operator. Make graphics (results, name labels, etc.) in real-time. Usually use a template and type in texts (Preview on the GRAF PW on the Screen 2, see Figure 5).

5) Graphic editorial. Check the graphics before sending them on air. Make and edit more complex graphics and commercial clips (preview on the TOG of the Screen 2, see Figure 5). 


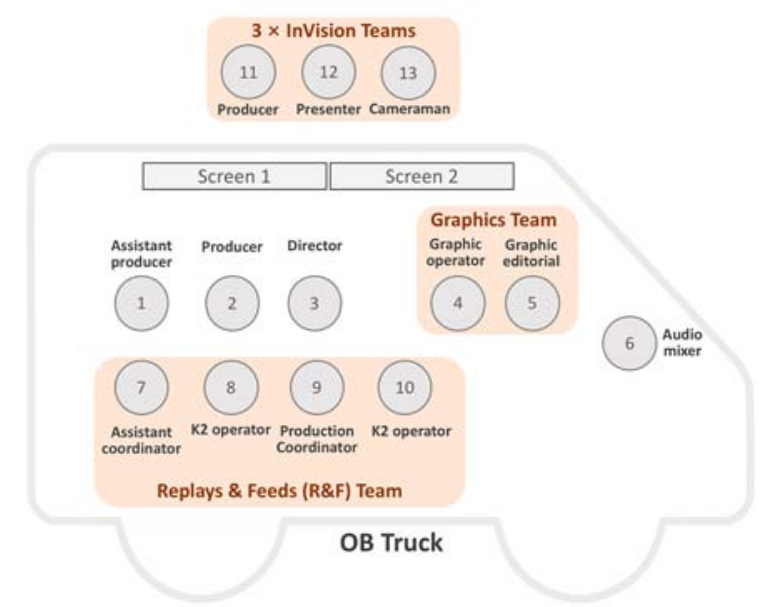

Figure 4. Roles of staff on an OB truck and the InVision teams on the tracks.

6) Audio mixer. When the program is on air, making sure it has the correct audio signal.

7) Assistant coordinator. Have a good knowledge of MotoGP, help production coordinator to decide on the making and selection of the replay clips.

9) Production coordinator. Direct the K2 operators to make replay clips (e.g., deciding when the clip starts and how long it should be).

8) \& 10) K2 operators. K2 is a replay making and archiving system. Two operators are constantly working with it during the live broadcasting.

In the OB truck, two 55-inch screens were placed in front of the director. The main preview screen is the Screen 2. A quarter of the screen was the actual live feed of the program on a TV screen. Another quarter of the Screen 2 was taken by the preview of the program. The rest of the Screen 2 was filled up by four preview channels (named by different colors as GREEN, PINK, BLUE, RED), the upcoming feeds and replays were placed in these four channels. The Screen 2 also had a mini-preview for graphics (GRAF PW), and the camera feeds from the three InVision teams. The Screen 1 had the same four channels (GREEN, PINK, BLUE, RED) for upcoming feeds and replays. One quarter of the Screen 1 was the "World Feed" from Dorna. The Screen 1 also exhibited the local time, helicopter feeds and commercial previews. Details of the two screens divisions are illustrated in Figure 5.

The workflow in the North One OB truck

The broadcasting work of North One Television is written down as a "running order document". The document consists of the pre-race warming ups, the in-race commentary, the replays and graphics in addition to Dorna's, and the post-race commentary, which is specified with a minute by minute schedule. The staff use the minutes as reference points throughout the live broadcasting process. The document also defines where graphics, visual sources, and sound come from and when they are "on-air". It contains the duration of each

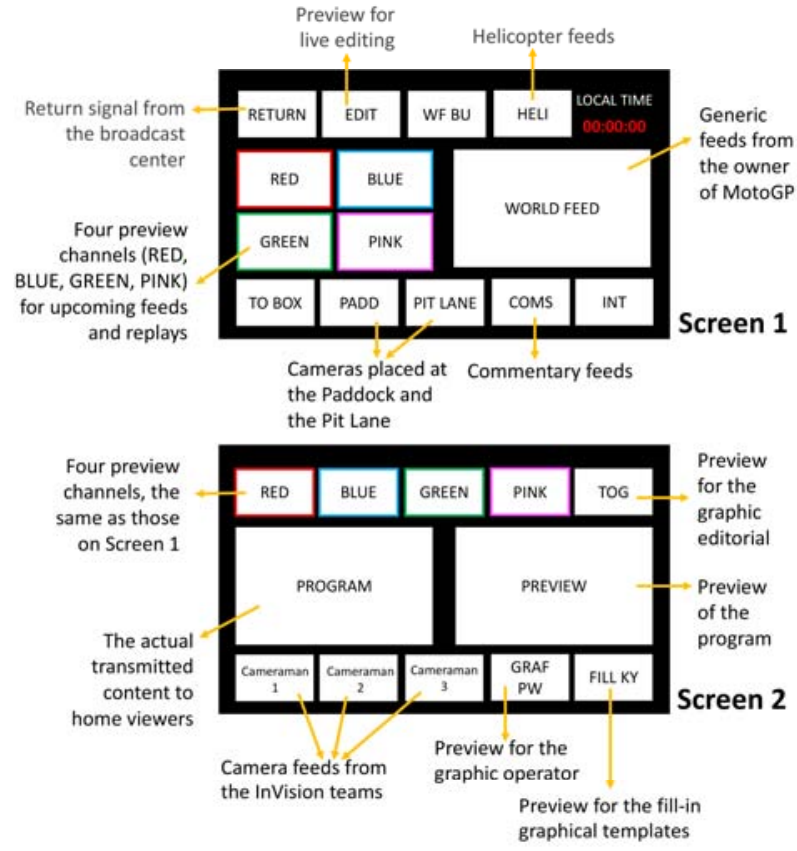

Figure 5. Two screens in front of the director.

item and the approximate time it starts. Table 1 presents a brief example of such a document. A program is segmented into many parts with 3-minute commercial breaks inbetween. During the breaks, the producer announced the sequence of content that would be aired in the coming period. The workflow within each part was the same. The main tasks involved in the workflow are visualized in Figure 6 and explained as follows:

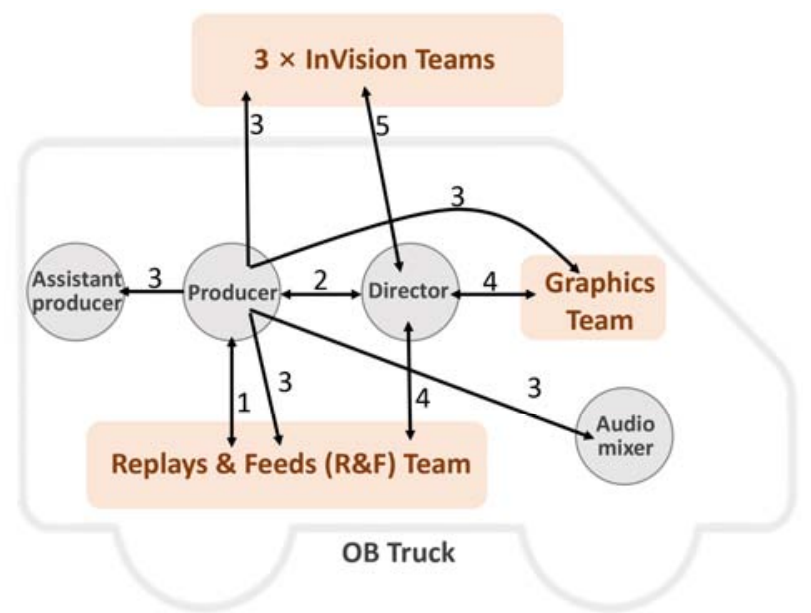

Figure 6. The workflow of the live broadcasting.

1) The producer communicates with the $R \& F$ team, collecting interesting materials to include in the broadcast. The R\&F team "sells" the interesting feeds and replays to the producer.

2) The director works closely with the producer, checking all feeds, and deciding together with the producer the sequence of events for the coming 10-15 minutes. 


\begin{tabular}{|c|l|l|l|l|c|c|}
\hline ITEM & \multicolumn{1}{|c|}{ GRAPHICS } & SOURCE & \multicolumn{1}{|c|}{ SOUND } & \multicolumn{1}{|c|}{ DESCRIPTION } & DURATION & TIME OF DAY \\
\hline & & & & WARM UP SHOW 5’00 & & 084500 \\
\hline $\mathbf{1}$ & Opening animation & VT & Bubble & Opening & $00: 00: 08$ & 084508 \\
\hline $\mathbf{2}$ & Presenter name & CAMS & PRES MICS & LIVE: Presenter welcome & $00: 00: 20$ & 084528 \\
\hline $\mathbf{3}$ & & VT & SOVT & VT: Welcome & $00: 00: 36$ & 084604 \\
\hline $\mathbf{4}$ & & CAMS/VT & $\begin{array}{l}\text { PRES } \\
\text { MICS/SOVT }\end{array}$ & $\begin{array}{l}\text { LIVE: Presenter interview } \\
\text { VT: Player introductions }\end{array}$ & $00: 03: 30$ & 084934 \\
\hline$\ldots$ & $\ldots$ & $\ldots$ & $\ldots$ & & $\ldots$ & $\ldots$ \\
\hline $\mathbf{n}$ & & & & OFF AIR-08:50:45 & & 0854 \\
\hline
\end{tabular}

(VT: Videotape; CAMS: Cameras; PRES MICS: Presenter microphones; SOVT: Sound of videotape.)

Table 1. An example of a "running order document" for live broadcasting.

3) The producer announces the decisions to the rest of the team.

4) When events happened, the director asks for particular feeds from the R\&F team and requests the graphics team to create specific graphics based on the templates.

5) The InVision teams may be requested by the director to shoot particular scenes or talk about particular topics.

\section{Challenges of live broadcasting}

During the live broadcasting, especially for such an intense sports event, the team members were utterly focusing on their own tasks. The workload of the producer, the director and the replay operators was extremely heavy. We believe that it would be difficult for the team to use current equipment to produce extra content for multiscreens. Thus, the next section proposes a multiscreen production platform, for both authoring and broadcasting live content to TV screens as well as companion screens.

\section{A MULTISCREEN PRODUCTION PLATFORM}

As introduced in the "background" section, a preproduction tool was designed and evaluated in previous studies. This section will present the design of the live editing tool. The two tools, namely the preproduction tool and the live editing tool, are two parts of the proposed multiscreen production platform.

\section{The Live Editing Tool}

The goal of the live editing tool is to enable the production team to do live broadcasting for multiscreens, to reduce their workload and to quickly react to events. Compared with the preproduction tool, the interface of the live editing tool is rather simple and straightforward. There are two tabs. The left tab (Figure 7a) contains all the components and events prepared in the preproduction tool (e.g., logos, name tags, live event replays etc.). The right tab (Figure $7 b$ ) contains the components and events being triggered at the moment. Under the right tab, the triggered components can be removed during live broadcasting. For instance, when the leaderboard takes too much space and hides something of interest, the director can decide to remove the leaderboard for the time being. Figure 7 exhibits some examples of the prepared and triggered components in the live editing tool, and a preview on a TV screen.
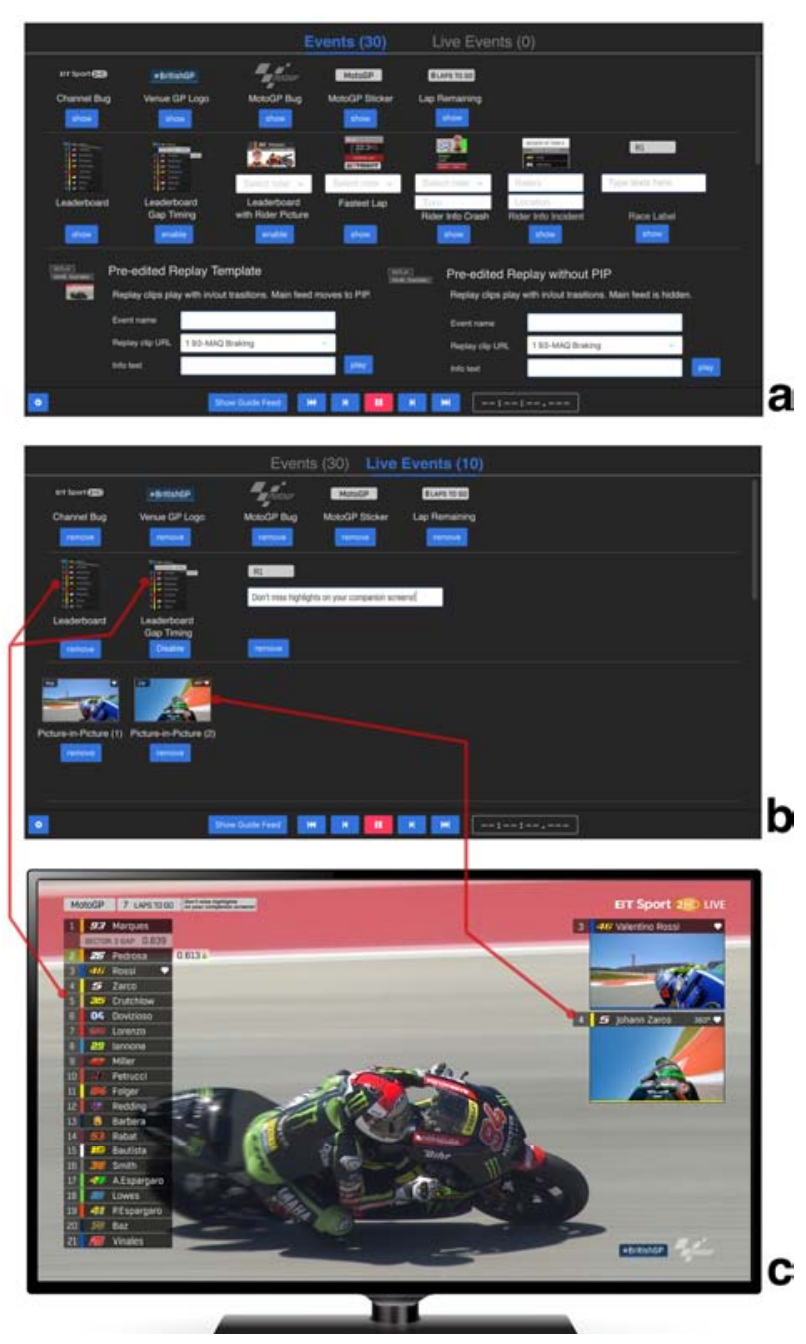

Figure 7. Some examples of (a) the prepared components and (b) triggered components in the live editing tool and (c) a preview on a TV screen.

\section{A proposed workflow}

The production platform proposes some changes to the typical TV production workflow (see Figure 2). The changes focus on the preproduction and the live editing parts, enabling the production team to create and broadcast for multiscreen, both for live and recorded content. The preproduction tool digitalizes the "running order document" 


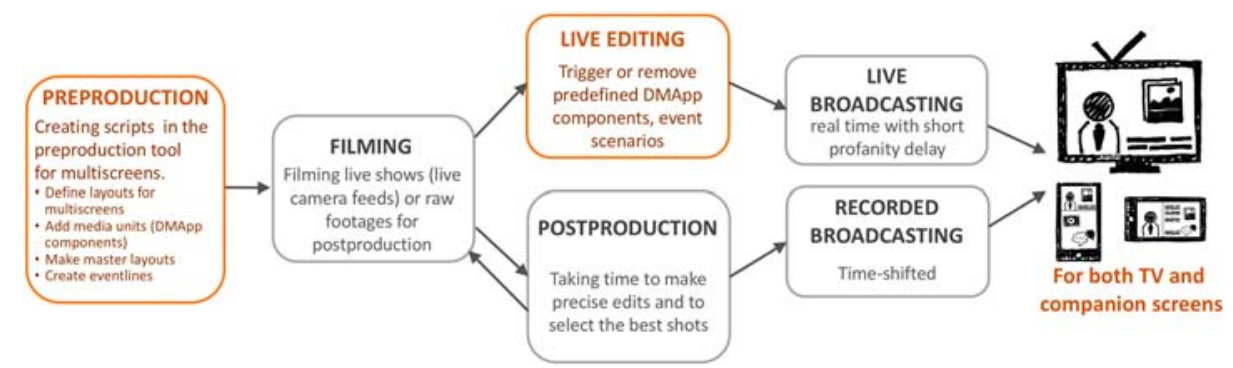

Figure 8. The changes (in orange color) made by the production platform to the typical television broadcasting workflow.

or script and allows the production team to prepare for anticipated live events (e.g., a crash in MotoGP or a goal in football). It also enables the team to preview the created experience on multiscreen without having a video feed. The live editing tool becomes simple since most of the work has been done at the preproduction phase. The team only needs to trigger the prepared components and live events at the right time. The editing task is simple. For instance, the team chooses a rider's name from a drop-down menu, and click on "show", the profile component will automatically generate the information about the rider and air it on TV or companion screens. The changes in the workflow are highlighted in Figure 8. The new set of tools for preproduction and live editing of a program are sketched in Figure 9.

To validate whether the workflow proposed by the production platform is appropriate, the next section presents validation interviews with professionals who are experienced in producing live interactive TV program, developing companion screen applications, doing research about new production workflows and so on.

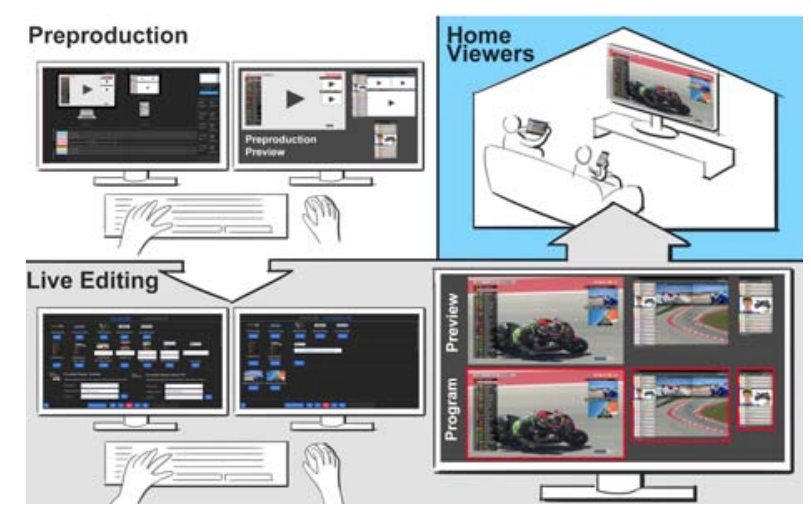

Figure 9. Preproduction and live editing of a program.

\section{VALIDATION INTERVIEWS}

The goal of the validation interviews is three folds. The first is to validate whether the proposed workflow for multiscreen production is appropriate (e.g., whether it fits into the current production workflow). The second is to collect suggestions from professionals for improving the tools. The third is to find out who is the right person in the production team to use such new tools.

\section{Methods}

Six semi-structured interviews [24] were conducted at the BBC usability lab, on Jan 18 and 19, 2018. This section introduces the selection of the interviewees, the procedure of the validation interviews, the data collection and the analysis.

\section{Interviewees}

Ten broadcasting professionals (P1-P10) with related backgrounds were invited to validate the workflow and the platform. They are either experienced in producing interactive TV, developing companion screen applications or doing research on multiscreen immersive TV experience and new production workflows (see Table 2). Most interviews were one-to-one conversations except two, which was conducted with two (P2 \& P3) and four professionals (P6, P7, P8 \& P9), due to their limited time availability.

\footnotetext{
P1 Interactive TV researcher at BT, researching on next generation multiscreen and immersive TV application, and experienced in UX design and evaluations.

P2 UX designer at BBC, developing companion screen applications and websites.

P3 Creative director at BBC, creating online content for TV programs.

P4 Senior UX designer at BBC, doing researching about workflows and roles in a production team.

P5 Computer scientist at BBC, researching on HCI and UX.

P6 TV series producer at BBC, producing live interactive TV programs and working in production studios

P7 Broadcast journalist and assistant producer at BBC, creating online and interactive content for TV programs.

P8 Assistant producer at BBC, creating online and interactive content for TV programs, working in production studios

P9 New workflow researcher at BBC, working with production studios, developing innovative tools for the production of subtitles and shot planning for multi-camera TV direction

P10 Creative director for production systems at BBC, developing and testing production tools
}

Table 2. Ten professionals and their daily work.

Procedure

The prototype of the production platform was installed at the usability lab. Two interviewers facilitated the interviews. The interviews lasted about one hour, mainly consisting of three steps as follows:

1) One interviewer introduced some related topics, including, among others, multiscreen viewing and object-based broadcasting. At the end of the introduction, the new workflow and the goals of the interviews were explained. 
2) With the interview goals in mind, the prototype of the production platform was played by one facilitator and presented to the professionals. The preproduction tool was shown first, then the live editing tool. Open discussions were encouraged around whether the new workflow is appropriate or not.

3) The professionals were requested to give improvement suggestions of the interface and indicate the suitable person(s) that can use the tool in the future.

\section{Data collection and analysis}

The interviews were video and audio recorded. The audio records were transcribed into text. The video records were used to clarify ideas during the transcription process. Relevant quotations from the transcripts were selected and coded according to the goals and the discussions of three researchers in the project. Figure 10a shows an example of the coded texts. It consists of a quotation from a professional (e.g., P3) selected from the transcripts and an interpretation of the researcher (the handwritten texts). The coded texts were then sorted into four main categories, namely positive aspects of the platform, ways to fit into the current production workflow, suitable staff to use the platform, and improvement suggestions. The results section presents the four main categories (Figure 10b).

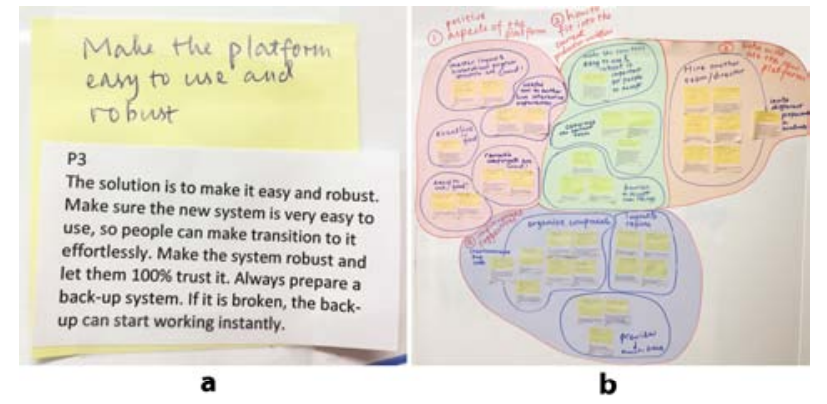

Figure 10. (a) One example of the coded texts; (b) The coded texts were sorted into four main categories.

\section{Results}

According to the research questions, the results were sorted into four main categories. The first category describes the positive aspects of the production platform complimented by the professionals. The second category gathers the comments about how to fit the platform into the current production workflow. The third one consists of suggestions for suitable staff to operate the platform. The last category presents the recommendations from the professionals for improvement. The four categories are presented in the following subsections.

\section{Positive aspects of the platform}

P1, P2 and P3 liked the master layout design and hierarchical overview of the program chapters, which reduce the repetitive work. They also indicated their preference for the re-usable DMApp components, since keeping a short list of re-configurable components can save the effort of searching. Another aspect they considered as appropriate is the "eventline" (P2, P3, P5 \&P10). Components and events are arranged in sequence on the eventline because a program is based on a sequence of components and events. For some events, especially the live ones, it is impossible to know their length during preproduction. P4 pointed out that the dark interfaces can bring a sense of familiarity to most production team members since they are frequent users of video editing tools. Most of these tools have a dark interface (e.g., Adobe Premiere). P4 also mentioned that the live editing tool is very "light", "it may not need a level of expertise to be able to use it", which is a good sign for the production team to be motivated to try it. P6, P7 and P8 thought the platform worked very much in parallel as their Live Lessons (a live education program). They saw opportunities to offer live interactive lessons to students. P6 also indicated an opportunity to use the platform for pre-recorded programs, but make it feel like a live interactive experience. As P6 explained, "It will be more cost-effective to do recorded broadcasting than live broadcasting, but we do not want to sacrifice viewers' live interactive experience."

\section{How to fit into the current production workflow}

The professionals had consistent opinions about how to fit the platform into the current production workflow. They believed that it might be difficult to replace the current workflow (P3, P4 \& P9). As P3 explained, "People tend to use the old-fashioned software. Once they get trained to use it, they do not want to change to new systems." "It might be quite some barriers to let people who work on TV screens switch to multiscreen (P9)."

To change this reluctance to adopt a new production platform, the professionals suggested two ways. The first is to "make it easy and robust" (P3). As P3 explained, "Make sure the new system is straightforward, so people can make a transition to it effortlessly. Make the system robust and let them $100 \%$ trust it. Always prepare a backup system. If it is broken, the back-up can start working instantly." The second is to convince the production team that multiscreen TV view is valuable (P3, P6 \& P9). "It will be good to let the production team to play with the platform, to try out different scenarios of multiscreen TV viewing experiences (P3)." "Specify scenarios of audience experiences. Some of them might have no companion screens but a TV screen, some of them might have a tablet, a phone and a TV, others might have two phones, and so on. Give the director a list of new audience experiences. Let him feel that this is new, and they want to try (P9)."

\section{Suitable staff to use the platform}

P2, P3, P4, P6, P7, P8, P9 and P10 all gave similar ideas about who will use the platform. "Practically, it makes more sense that a separate team or a person to be trained to operate this platform. It lacks space in the current production team, to really work for companion screens (P3)." P4 suggested that "It seems to me that this should be another team working on this platform, a team with a focus on social, online and companion content, not on TV (P4)." P6 and P9 recommended a new role called "an interactive 
director" or "an interactive direct team", to use this platform. P2 and P10 further specified that people within this interactive team should also clearly divide the tasks. "For example, a person decides to show or to remove components, and another person decides where the components go, to companion screens or to TV screens (P10)." P10 also suggested inviting different production teams (e.g., small or big, different genres) to use the platform. For example, a small and young production team that makes relatively simple program might adopt this new system more easily than the well-established and sophisticated production teams.

\section{Improvement suggestions}

Improvement suggestions were mainly about the interface design. A few professionals questioned about the layout mechanism in the preproduction tool (P2, P3 \& P10). They were confused about the regions and overlays of components. Frequently asked questions include "Does the content take the entire space of the region it assigns to (P2)?"; "Is there a way to specify the exact positions of certain components, to prevent they overlap undesirably (P10)?"; "How to arrange components that are placed in the same region (P3)?" In the end, three modes of layout design were proposed based on the feedback from the professionals. The first mode is the easy mode, where a library of templates is prepared. The second mode is region mode, where TV screens and companion screens are divided into several regions. Every region can only hold one component at a time, and the component will take up all the space in that region. Components in different regions will not be overlapped. The third mode is the advanced mode, where the exact positioning of every component can be specified. The main video stream is overlaid by all the components. Figure 11 shows the region mode and the advanced mode.

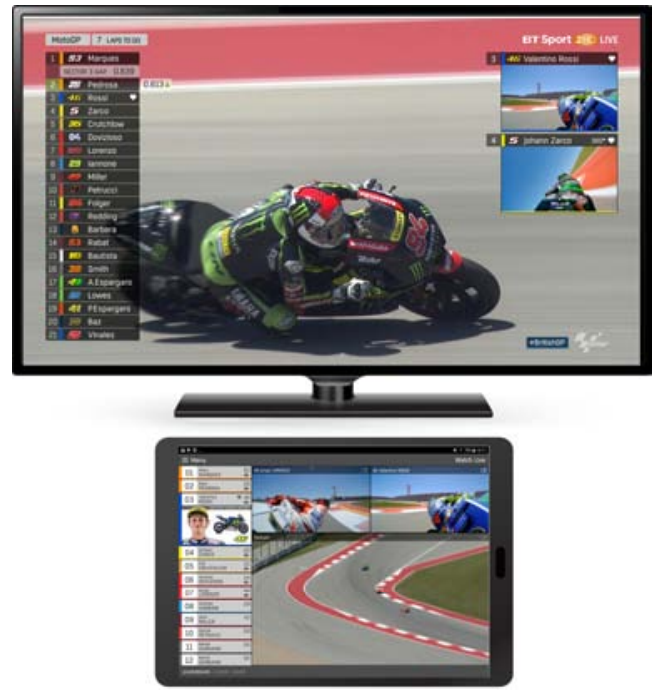

Figure 11. Advanced mode of layout design for a TV screen, where the video stream is overlaid by components with accurately specified positions (above); region mode of layout design for a tablet screen, where a screen is separated into multiple regions (below).
Another consistent feedback is about organizing the components in the live editing tool (P1, P4, P5, P6, P7, P8 \& P9). The professionals complained that the list of the prepared components is very long with a lot of scrolling to find a component. P10 suggested thinking about several drop-down lists instead of a scrolling list. Within each dropdown list, there can be selections of components that can be grouped together, such as the icons, logos and statistic graphics are under one list. Name tag templates and statistics templates are under another list.

The professionals also indicated that the two tabs in the live editing tool are counterintuitive (P6, P7 \&P8). People need to constantly switch between two tabs in order to trigger or stop certain components. They suggested to use only one button for triggering and stopping a component, like a switcher for light: click once to trigger and click again to stop. Adding preview to the live editing tool was also requested by three professionals (P2, P4 \& P10). "You do not need to show all the content in the preview on a big screen. A mini-preview that gives ideas about the position of the triggered component is sufficient.

\section{DISCUSSION AND CONCLUSION}

This paper proposes a production platform and a new workflow for authoring and broadcasting TV programs to multiscreen. The platform was validated by ten professionals in the TV broadcasting industry. They indicated that the user interfaces of the platform are straightforwardly designed and allows well preparations at the preproduction stage and reduce the workload during the live broadcasting. They foresaw the potential of the platform in producing multiscreen contents for both live and recorded broadcasting and provided feedback to improve the interface design, such as clarifying the layout design mechanisms in the preproduction tool, organizing components and adding previews in the live triggering tool. The study process has some limitations. For instance, during the field study at Silverstone, we did not have opportunities to have a conversation with the production team while they were working. Some valuable insights may be neglected. The prototype was not fully implemented, so it was impossible to ask the professionals to operate the platform during the interviews. The future evaluations will be conducted with a fully developed platform in real live broadcasting context.

Apart from the limitations, the validation interviews provided useful insights for the redesign and implementation of the platform. The professionals specifically pointed out several aspects that need be carefully considered, such as how to make the digitalized "running order documents" as flexible as the paper documents. In other words, when something happened during live broadcasting, the team needs to quickly delete some pre-authored content and quickly build new content to replace it. Another aspect is about whether multiple members of the team can work on the platform together. In this case, the task dividing among team members is important, to avoid conflict in editing. The third 
aspect is to think about the "responsive design" like a website. The authored content can automatically rescale to fit into different sizes of screens; or the same content has different modes (e.g., novice versus expert) that are customized to personal interest. The viewers have some flexibility in selecting modes or resolutions. The production team should have the editorial control (e.g., decide the recommended screen size for a program, and decide how many modes a program has). They indicated that the current production team needs to hire extra staff to use the platform. The platform can run in parallel with the existing production workflows. However, it might require time and effort to get the production team to accept and trust a new system.

Both end viewers and production companies are believed to benefit from the proposed new platform. For end viewers, the platform offers interactive contents and personalized and immersive watching experiences. For production companies, the platform helps reduce the workload of live broadcasting, making authoring and broadcasting possible for multiscreen. The next step of the project is to accomplish the development of the production platform and test it at a stadium, for real live football broadcasting. Meanwhile, it will be tested with end viewers to evaluate their experiences. The tests will happen in the summer of 2018.

\section{ACKNOWLEDGEMENT}

This work is supported by European Union's Horizon 2020 research and innovation program under grant agreement No 687655, project 2-IMMERSE.

\section{REFERENCES}

1. Edward Anstead, Steve Benford, and Robert J. Houghton. 2014. Many-screen viewing: evaluating an Olympics companion application. In Proceedings of TVX '14, 103-110.

2. Mike Armstrong, Matthew Brooks, Anthony Churnside, Michael Evans, Frank Melchior, Matthew Shotton. 2014. Object-based broadcasting- curation, responsiveness and user experience. In Proceedings of IBC 2014 Conference, Amsterdam, The Netherlands, p. 12.2.

3. BBC Research \& Development. 2015. Squeezebox: A production tool that can edit for you. Retrieved December 5, 2017, from http://www.bbc.co.uk/rd/projects/squeezebox

4. Frank R. Bentley. 2017. Understanding Secondary Content Practices for Television Viewing. In Proceedings of TVX '17, 123-128.

5. Pablo Cesar. 2015. From secondary screens to sociallyaware and immersive experiences (invited talk). In María José Abásolo, Francisco J. Perales and Antoni Bibiloni (Eds.), Applications and Usability of Interactive $T V$, p. VII-VIII.
6. Jasmine Cox, Rhianne Jones, Chris Northwood, Jonathan Tutcher, and Ben Robinson. 2017. ObjectBased Production: A Personalised Interactive Cooking Application. In Adjunct Publication of TVX '17, 79-80.

7. Mihaly Csikszentmihalyi and Robert Kubey. 1981. Television and the rest of life: A systematic comparison of subjective experience. Public Opinion Quarterly, 45(3), 317-328.

8. John Dowell, Sylvain Malacria, Hana Kim and Edward Anstead. 2015. Companion apps for information-rich television programmes: representation and interaction. Personal and Ubiquitous Computing, 19(7), 12151228.

9. K. Anders Ericsson and Herbert A. Simon. 1980. Verbal reports as data. Psychological Review, 87 (3), 215-251.

10. David Geerts, Rinze Leenheer, Dirk De Grooff, Joost Negenman, and Susanne Heijstraten. 2014. In front of and behind the second screen: viewer and producer perspectives on a companion app. In Proceedings of TVX '14, 95-102.

11. Christian Holz, Frank Bentley, Karen Church, and Mitesh Patel. 2015. "I'm just on my phone and they're watching TV": Quantifying mobile device use while watching television. In Proceedings of TVX '15, 93102.

12. Jianmin Jiang, Joachim Kohler, Carmen Williams, Janez Zaletelj, Georg Guntner, Heike Horstmann, Jinchang Ren, Jobst Loffler, and Ying Weng. 2011. "Live: An integrated production and feedback system for intelligent and interactive TV broadcasting." IEEE Transactions on Broadcasting, 57(3), 646-661.

13. Hans Jonasson. 2012. Determining Project Requirements. ESI International Project Management Series. CRC Press.

14. Holtzblatt, K. and Beyer, H., 2014. Contextual design: evolved. Synthesis Lectures on Human-Centered Informatics, 7(4), pp.1-91.

15. Ian Kegel, James Walker, Mark Lomas, Jack Jansen and John Wyver.2017. 2-IMMERSE: A platform for orchestrated multiscreen entertainment. In Adjunct Publication of TVX '17, 71-72.

16. Jie Li, Zhiyuan Zheng, Britta Meixner, Thomas Röggla, Maxine Glancy and Pablo Cesar. 2018. Design an Object-based Preproduction Tool for Multiscreen TV Viewing. Accepted by CHI 2018, late-breaking work.

17. Britta Meixner, Maxine Glancy, Matt Rogers, Caroline Ward, Thomas Röggla and Pablo Cesar, P., 2017, June. Multi-Screen Director: a New Role in the TV Production Workflow?. In Adjunct Publication of TVX '17, 57-62.

18. Janet Murray, Sergio Goldenberg, Kartik Agarwal, Tarun Chakravorty, Jonathan Cutrell, Abraham Doris- 
Down, and Harish Kothandaraman. 2012. Story-map: iPad companion for long form TV narratives. In Proceedings of EuroITV '12, 223-226.

19. Abhishek Nandakumar and Janet Murray. 2014. Companion apps for long arc TV series: supporting new viewers in complex storyworlds with tightly synchronized context-sensitive annotations. In Proceedings of TVX '14, 3-10.

20. Timothy Neate, Matt Jones, and Michael Evans. 2015. Mediating Attention for Second Screen Companion Content. In Proceedings of CHI '15, 3103-3106.

21. The Nielson Company. 2017. The Nielson total audience report Q1 2017. Copyright (C) 2017 The Nielsen Company. Retrieved December 5, 2017 from http://www.nielsen.com/us/en/insights/reports/2017/the -nielsen-total-audience-report-q1-2017.html

22. Carlos Antonio Navarrete Puentes and José Tiberio Hernández Peñaloza. 2017. HEd: A Flexible HbbTV
WYSIWYG Visual Authoring Tool. In Adjunct Publication of TVX '17, 15-20.

23. Jim Ownens and Gerald Millerson. 2009. Television production (The 15th edition). Focal Press.

24. Marina Remington and P. Tyer. 1979. The social functioning schedule: A brief semi-structured interview. Social Psychiatry, 14, 151-157.

25. Pedro Silva, Yasmin Amer, William Tsikerdanos, Jesse Shedd, Isabel Restrepo, and Janet Murray. 2015. A Game of Thrones Companion: Orienting Viewers to Complex Storyworlds via Synchronized Visualizations. In Proceedings of TVX '15, 167-172.

26. Doug Williams, John Wyver and Maxine Glancy. 2016. Evaluating the potential benefits of object-based broadcasting. Retrieved December 5, 2017 from https://nem-initiative.org/wpcontent/uploads/2016/11/doug-willams-obb.pdf 